\title{
Polymer/Layered Silicate Nanocomposite as Matrix for Bioinsecticide Formulation
}

\author{
Diogo P. C. Batista,${ }^{1}$ Rafael da S. Souza,${ }^{1}$ Nereide S. Santos-Magalhães,${ }^{2}$ José G. de \\ Sena-Filho ${ }^{3}$ Adenir V. Teodoro ${ }^{3}$ Luciano A. M. Grillo, ${ }^{1}$ Camila B. Dornelas ${ }^{* 1}$
}

Summary: Due to current encouragement to the use of bioinsecticides for pest control and the susceptibility of biological agents to external factors, we investigated the use of a polymer nanocomposite (PLN, polymer/ layered silicate nanocomposite) as matrix to encapsulate an entomopathogenic fungus active against pest insects of palm trees. The beads were formed by extrusion and the following variables were assessed: fungus conidial concentration (series $1: 10^{7}$; series $2: 10^{8}$ and series 3: $10^{9}$ conidia/mL) and nanolayered silicate concentration (0; $0.5 ; 1 ; 2$ and $4 \%$ ). The matrix was evaluated by X-ray powder diffraction and Fourier transform infrared spectroscopy and the following characteristics of the products were assessed: percent of encapsulated conidia, size distribution and polydispersity index, swelling index, formulation's in vitro ability to release conidia and stability under different storage temperatures. PLN, whose interactions could be visualized by FTIR, proved to be a potential matrix for this fungus, because, while composed by natural substances non-toxic to the environment, it succeeded to encapsulate high amounts of conidia (series 2). A barrier effect with bentonite increase was also demonstrated by increased fungus germination time and thermal stability.

Keywords: bioinsecticide formulation; entomopathogenic fungus; hydrophilic polymers; nanocomposites; silicas

\section{Introduction}

Pest-insects are a constant threat and a challenge to the farmer. The use of conventional chemical insecticides fatally leads to biological imbalances by killing useful insects such as pollinators and natural pest controllers, besides being hazardous to the handler. Thus, the status of agricultural pests in general is worsened in the years subsequent to the use of these products. ${ }^{[1]}$ Therefore, the Environmental Protection Agency increasingly encourages the use of

${ }^{1}$ Curso de Farmácia (Esenfar), Universidade Federal de Alagoas (UFAL), Campus A. C. Simões, Maceió, AL, Brazil

E-mail: dornelascb@yahoo.com.br

2 Laboratório de Imunopatologia Keizo Asami (LIKA), Universidade Federal de Pernambuco (UFPE), Recife, PE, Brazil

${ }^{3}$ Empresa Brasileira de Pesquisa Agropecuária (Embrapa Tabuleiros Costeiros), Aracaju, SE, Brazil biological pesticides or biopesticides to control crop pests. They generally do not affect the environment or human and animal health or species different from the target pest. ${ }^{[2]}$ Bioinsecticides based on entomopathogenic fungi are widely used with that purpose, however, fungus conidia are very sensitive to external factors (abiotic), thus their encapsulation is desirable to protect the fungal matrix and maintain the integrity of its main characteristics. $^{[3]}$ Still, the lack of practicality and reduced shelf life of the products based on entomopathogenic fungus available, hinder its marketing and restrict the potential use of these microbiological agents for Integrated Pest Management (IPM), encapsulation in polymer matrices adds value to the product. These formulations present better properties than non-formulated products and are essential to keep microorganism viability. They are also important for 
bioinsecticide standardization, thus enabling their marketing. ${ }^{[4]}$ Alginate is a biodegradable hydrophilic polymer, relatively inert, biocompatible, gelling agent, with adhesive properties, which are desirable characteristics for insecticide formulation, although it shows poor mechanical strength. ${ }^{[5]}$ Nanotechnology has been a resource considered to solve this problem. Nanostructured materials are those materials that have, in at least one direction, dimension in the nanometer range, which gives them unique characteristics. ${ }^{[6]}$ They may have all the crystallites and interface boundaries with the same chemical composition or not, resulting in structures known as nanocomposites. Polymer nanocomposites are two-phase materials, with one phase formed by nanoparticles (fillers) dispersed in a polymer matrix, which is the continuous phase. ${ }^{[7]}$ Different nanofillers may be incorporated into the polymer matrix; when the filler is a philosilicate clay such as bentonite, the material is generically called Polymer/ Layered Silicate Nanocomposite (PLN). These lamellar silicates present crystalline lamellae in the nanometric scale which are 2D arranged on top of each other. ${ }^{[8]}$ Bentonite increases the mechanical properties ${ }^{[9]}$ and has a photoprotective effect against UV radiation described in literature. ${ }^{[10]}$ Thus, the present study aims to research the use of the alginate-bentonite polymer nanocomposite as encapsulation matrix of an entomopathogenic fungus in a bead-type formulation.

\section{Experimental Part}

\section{Preparation of Conidial Suspensions}

Fungal cultures were obtained by plating a fungal isolate provided by Embrapa Tabuleiros Costeiros (CPATC032) on Petri dishes containing PDA (Potato-dextroseagar) nutrient medium (Himedia, India). The plates were kept in an oven (Solab, Brazil) at $26^{\circ} \mathrm{C}$ for 7-10 days and 12-hour photoperiod. The suspensions used in the experiments were prepared by scraping fungus colonies with a platinum loop with
L-shaped tip and inoculating in $0.02 \%$ solution of Tween 80 (Sigma-Aldrich, Germany) in distilled water. These suspensions were filtered through sterile gauze to remove the mycelium and conidial concentration was determined in a Neubauer's chamber (hemocytometer) (New Optik, Brazil) using an optical microscope (MetrimpaxHungary/PZO-Labimex, Poland). ${ }^{[1,12]}$

\section{Suspension Viability}

The viability of the suspensions obtained was assessed by seeding $150 \mu \mathrm{L}$ aliquots in Petri plates containing $5 \mathrm{~mL}$ of PDA culture medium and incubating at $28( \pm 1){ }^{\circ} \mathrm{C}$ and 12-hour photophase. After a 17 -hour period, the plates were withdrawn and the conidia observed with the aid of an optical microscope with a 40x objective. Conidium viability was determined by counting a total of 200 conidia in each plate which were classified as viable and non-viable. Conidia with germ tube length equal or greater than the conidium diameter were considered viable. ${ }^{[13]}$

\section{Preparation of Bioinsecticide Formulations}

The beads were produced through ionotropic gelation by extrusion of the suspension obtained by mixing the respective materials after 1-hour stirring at room temperature in $0.25 \mathrm{M}$ calcium chloride solution (Vetec, Brazil). ${ }^{[4]}$ The assessed variables were conidial concentration: $10^{7}$ (series 1$), 10^{8}$ (series 2) and $10^{9}$ conid$\mathrm{ia} / \mathrm{mL}$ (series 3); and bentonite (Bentec Laviosa Chimica Mineraria S.p.A., Italy) concentration: $0 ; 0.5 ; 1 ; 2$ and $4 \%$. Alginate (Sigma-Aldrich, Germany) concentration was fixed in $1 \%$. The beads were separated by filtration through filter paper, rinsed 3 times with deionized water and dried in vertical laminar flow hood (Filterflux, Brazil). ${ }^{[1]}$ Beads without fungus (blank) were obtained for matrix assessment. All the experiments were performed in triplicate.

\section{Matrix Assessment}

The matrix (polymer nanocomposite) was assessed by X-ray powder diffraction 
(XRPD) and Fourier transform infrared (FTIR) techniques as described below.

\section{$X R P D$}

The X-ray diffraction analyses were conducted in a DRX 3000 Shimadzu (Kyoto, Japan) diffractometer using $\mathrm{Cu} \mathrm{K} \alpha$ radiation at $30 \mathrm{kV}, 30 \mathrm{~mA}$. The powdered samples were analyzed at $0.02^{\circ}(2 \theta)$ intervals in the range from 3 to $90^{\circ}$. Bragg's equation was used to calculate lamellar clay basal spacing. ${ }^{[14]}$

\section{FTIR}

Infrared spectra were recorded on a Shimadzu Fourier transform spectrometer (SSU-8000) in mode diffuse reflection infrared Fourier transform spectroscopy (DRIFT).

\section{Bead Assessment}

The beads were assessed in relation to average diameter and polydispersity index (PI), encapsulation efficiency (EE\%), determination of the swelling index ( $\mathrm{Si} \%)$, in vitro release and thermal stability.

\section{Determination of Average Diameter}

The size of the beads was determined by measuring Feret diameter through digital image analysis, using an Olympus $b \times 51$ (Olympus, Japan) optical microscope with 10x magnification, coupled with a camera for digitalizing the images that were then analyzed by the DP2-BSW software. The average diameter was obtained by calculating the mean \pm standard deviation of 300 beads. In this occasion, bead micrographs were obtained to observe bead shape and optical density. The results obtained were also used to determine the PIs of the samples, by calculation using equation 1 below, ${ }^{[15]}$ where $\mathrm{D}_{0.9}, \mathrm{D}_{0.1}$ and $\mathrm{D}_{0.5}$ are, respectively, the distribution that corresponds to 90,10 and $50 \%$ of each sample.

$\mathrm{PI}=\frac{D_{0.9}-D_{0.1}}{D_{0.5}}$
$E E \%$

It was indirectly obtained from the water resulting from the washings during the bead preparation process (to choose which of the series 1,2 or 3 would continue the assessment); or directly, counting fungal conidia in the Neubauer's chamber (hemocytometer) after bead breaking in $0.1 \mathrm{M}$ phosphate buffer solution $(\mathrm{pH} 7.4) .{ }^{[12]}$

\section{Si\%}

50 beads of each formulation were separated and weighed to obtain the average weight. Then, the same sample was immersed in distilled water for 12 hours, gently dried and re-weighed to obtain the swelling index $(\mathrm{Si} \%)$ by equation 2 , where Wi is the weight of the beads after swelling, Ws is the weight of the dried beads. ${ }^{[16]}$

$\mathrm{Si} \%=\frac{W i-W s}{W s} \times 100$

\section{In Vitro Release Studies}

10 beads were plated in PDA, randomly distributed on the entire plate. Fungal growth was monitored by visual observation of mycelium germination. ${ }^{[17]}$

\section{Stability Studies}

Bead samples were stored refrigerated $\left(4 \pm 1^{\circ} \mathrm{C}\right)$ and at room temperature and were assessed over a 7-month period, and their viability was observed by plating 10 beads of each formulation in culture medium. The beads that showed mycelium germination were classified as viable. ${ }^{[17]}$

\section{Statistical Analysis}

Variance analysis (Anova) was applied to the data obtained through GraphPadPrism 5.00 program.

\section{Results and Discussion}

\section{Fungal Suspensions}

The viability tests resulted in high germination percent for all the suspensions used in the experiments, 96.8 ; 96.7 and 95.3 for the 
series $1 ; 2$ and 3 , respectively. Values higher than $80 \%$ are usually regarded as ideal for the infective activity of entomopathogenic fungus. ${ }^{[18]}$

\section{Matrix}

The diffractograms of the polymer nanocomposite and respective starting materials are shown in Figure 1. The same diffractograms profiles were obtained for all the matrix formulations (blank), and the formulation with the highest bentonite concentration (AB4) was chosen to illustrate.

As expected, since alginate is an amorphous material, ${ }^{[19]}$ its diffractogram did not present crystalline planes. Bentonite showed d001 basal spacing of $15.26 \AA$ $\left(2 \theta=5.8^{\circ}\right)$. The matrix diffractograms did not present displacement of the bentonite original d001 plane, i.e., there was no alteration of the basal spacing, indicating that there was no intercalation. When the structure is exfoliated, clay diffraction peaks disappear, because the crystallographic shape is lost, which was also not the case. ${ }^{[6]}$ Kevadiya et al., ${ }^{[20]}$ found the same result, and suggested that the alginate interacts with bentonite through bonds with the hydroxyl groups on the surface of the montmorillonite (MMT), major constituent of this clay, forming intermolecular and electrostatic hydrogen bonds. This result on a $3 \mathrm{D}$ network with several contact points between both compounds. It was also suggested that alginate chains act as a bridge linking neighbor silicate layers when present in high concentration. We tried to elucidate this assumption using the FTIR technique.

FTIR spectra of alginate, bentonite and matrix formulation (blank) are shown in Figure 2. Asymmetric and symmetrical elongation vibrations at 1615 and $1417 \mathrm{~cm}^{-1}$ are due to carboxylic anions, and $1030 \mathrm{~cm}^{-1}$ for cyclic ether bridge oxygen stretching of alginate. ${ }^{[20]}$ The MMT shows bands from 3420 to $3898 \mathrm{~cm}^{-1}$, which are due to the high number of hydroxyl groups bonded to silica and aluminum $(\mathrm{OH}-\mathrm{Si}$ and $\mathrm{OH}-\mathrm{Al})$ present in the clay surface, generating high amplitude peaks, similar to those found in alginate structure, which has hydroxyl groups derived from the alcoholic groups of its molecular structure. Peaks derived from clay $\mathrm{Si}-\mathrm{O}$ structure are seen at 1015 $\mathrm{cm}^{-1}$ and peaks in the region of $1633 \mathrm{~cm}^{-1}$ is attributed to $-\mathrm{OH}$ bending mode of adsorbed water. ${ }^{[21]}$ In the matrix spectrum, there was a shift of $\mathrm{COO}^{-}$vibrations of alginate to a higher wavenumber (1606 and $1422 \mathrm{~cm}^{-1}$ ) and the intensity decreases. The modification observed in its cyclic ether peaks (1030 to $1024 \mathrm{~cm}^{-1}$ ) might have been caused by the interaction between alginate and bentonite which results in intensity increasing. Thaned et al., ${ }^{[2]}$ also reported
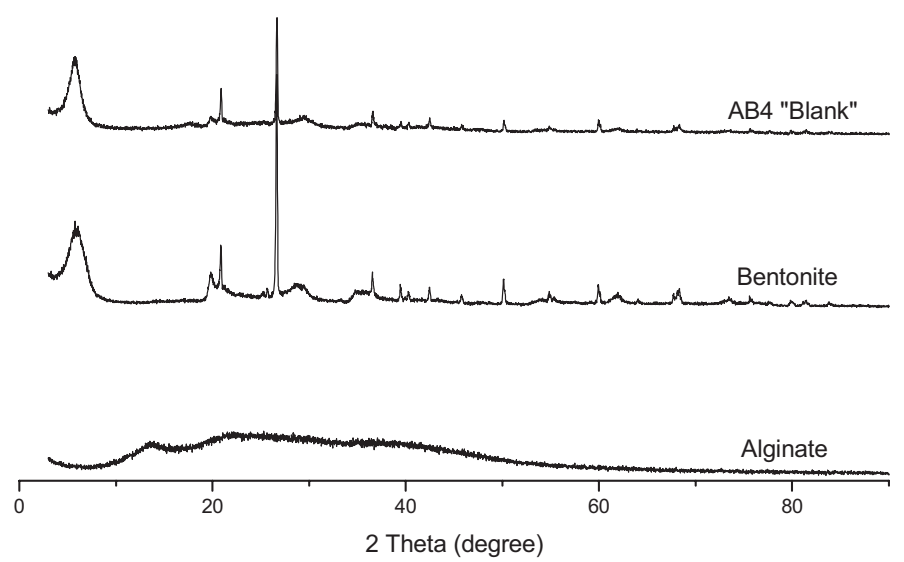

Figure 1.

XRD spectra of alginate, bentonite and the $\mathrm{AB} 4$ formulation. 


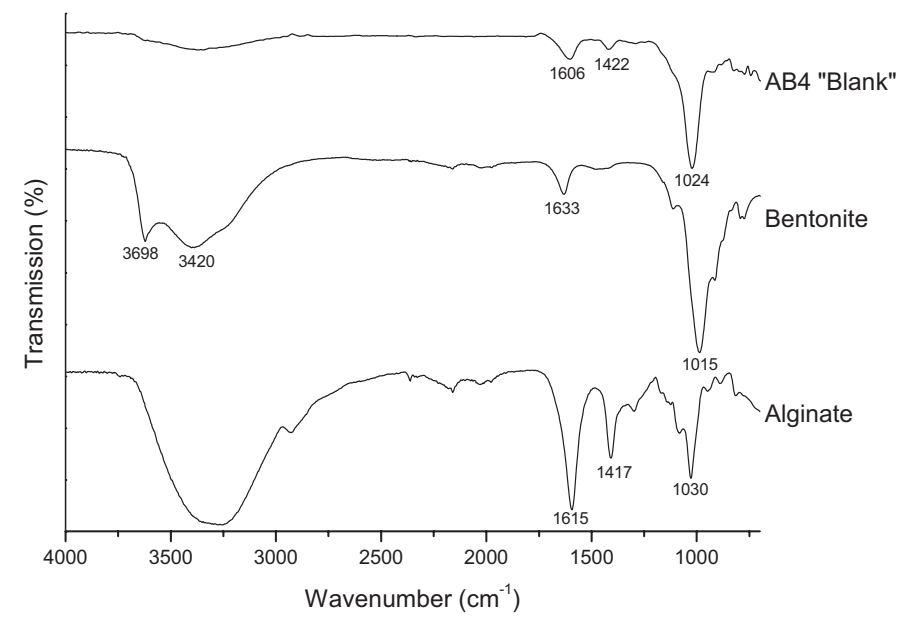

Figure 2.

FTIR spectra of alginate, bentonite and the $A B 4$ formulation.

this phenomenon. Negatively charged alginate carboxyl groups could interact with positively charged sites on MMT edges. According to Kevadiya et al., ${ }^{[20]}$ peak intensity increase in the hydroxyl region indicates the occurrence of intermolecular hydrogen bonds, which increase the electrostatic forces between those two compounds. Thus, those intermolecular and electrostatic hydrogen bonds cause numerous contact points for the creation of the 3D network and prove the formation of polymer nanocomposite.

\section{Beads}

When the fungal conidia were added, the matrix proved to be able to encapsulate them, even though at the highest concentration assessed, with indirect yield higher than $90 \%$. The reticulation of the mixture fermented biomass/polymer nanocomposite in calcium chloride generated firm, touch resistant and well defined beads in the series 1 and 2. However, in the series 3, where a higher conidium concentration was used $\left(10^{9}\right.$ conidia $\left./ \mathrm{mL}\right)$, the beads were more fragile, even getting to coalesce, regardless the silicate concentration assessed. This result was also observed by Pasqualim et al., ${ }^{[23]}$ who reported the deformation of polymer microparticles when the encapsulation capacity of the material was exceeded. The series 2 was then chosen to continue the formulation research, because it was the series that allowed the encapsulation with higher concentration of fungus conidia without affecting the product shape.

Silicate addition caused differences in bead mechanical properties: they appeared firmer and more well-defined when the silicate concentration increased, and they were more easily formed during dripping.

Figure 3 shows the size distribution curves of the beads obtained from series 2 , and we can observe that the size range slightly varied among the different formulations, between 650 and $1150 \mu \mathrm{m}$, similar to other reports in literature where similar matrices were used. $\mathrm{Wu}$ et al., ${ }^{[4]}$ found particle sizes between 980 and $1300 \mu \mathrm{m}$ using a matrix composed of alginate, bentonite and starch for bacteria encapsulation. However, Cabellero et al., ${ }^{[24]}$ when encapsulating ibuprofen in alginate microspheres by the ionotropic gelation technique, found a wider size distribution range, from 700 up to $1600 \mu \mathrm{m}$.

The values of bead average diameter obtained, as well as the PI were very similar. AB4 formulation presented the smallest average diameter and smallest PI. These values are shown in Table 1 that lists the 


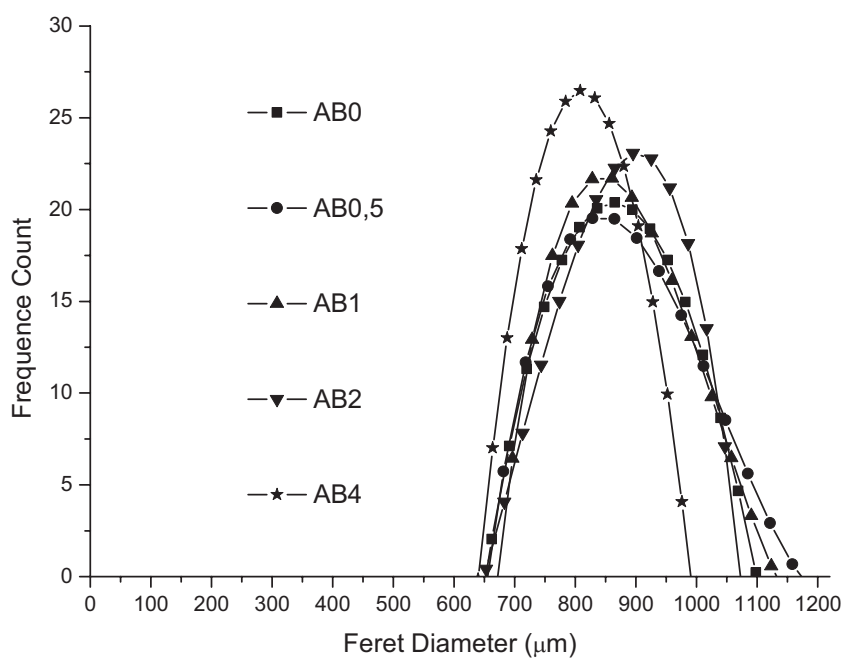

\section{Figure 3.}

Frequency of bead distribution of different silicate concentrations in series 2 .

parameters obtained for all the formulations of this series. All the products presented narrow size distribution or granulometric range $(\leq 0,3)$, i.e., they were homogeneous.

The micrographs (Figure 3-detail) illustrate an increase in optical density and spherical shape of the beads proportional to the increase of silicate concentration in the formulation.

EE\% was higher than $50 \%$ for all the formulations, proportionally increasing with clay mineral addition up to $2 \%$ concentration. This result showed better encapsulating properties of the silicateenriched polymer matrix, with better use of the active ingredient. Hydrophilic biopolymers have the ability to form gel, retaining high amounts of water inside, without dissolution occurrence. They are widely used in the pharmaceutical formulations and have been widely applied in encapsulation techniques. However, these gels present high porosity, and can cause high diffusion indices of the active principle. Silicate addition increased this characteristic, according to the results of size and swelling degree of the beads, which had smaller $\mathrm{Si} \%$ as silicate concentration increased. This type of matrix systems, when in contact with dissolution media, can present alterations of water capture capacity, whether by changes of the diffusion rate or by modifications of the nanocomposite structural framework. ${ }^{[16]}$

The result was confirmed through the observation of the time needed for mycelial germination, illustrated in Figure 4. It took 24 hours for its occurrence at $0.5 ; 1$; and $2 \%$ silicate concentration and in its absence.

Table 1.

General characteristics of different bead formulations.

\begin{tabular}{lcccccc}
\hline Formulation & $\begin{array}{c}\text { Alginate } \\
(\% \mathrm{~m} / \mathrm{v})\end{array}$ & $\begin{array}{c}\text { Bentonite } \\
(\% \mathrm{~m} / \mathrm{v})\end{array}$ & $\begin{array}{c}\text { Average } \\
\text { Diameter } \\
(\mu \mathrm{m})\end{array}$ & $\begin{array}{c}\text { Polydispersion } \\
\text { Index }\end{array}$ & $\begin{array}{c}\text { Encapsulation } \\
\text { efficiency } \\
(\%)\end{array}$ & $\begin{array}{c}\text { Swelling } \\
\text { Index } \\
(\%)\end{array}$ \\
\hline $\mathrm{AB0}$ & 1.0 & - & 869.8 & 0.29 & 51 & $70.0 \pm 4.6$ \\
$\mathrm{AB0} .5$ & 1.0 & 0.5 & 883.0 & 0.30 & 56 & $55.7 \pm 3.0$ \\
$\mathrm{AB} 1$ & 1.0 & 1.0 & 875.6 & 0.27 & 73 & $54.7 \pm 2.1$ \\
$\mathrm{AB} 2$ & 1.0 & 2.0 & 881.7 & 0.23 & 88 & $52.3 \pm 2.3$ \\
$\mathrm{AB} 4$ & 1.0 & 4.0 & 811.5 & 0.24 & 81 & $41.0 \pm 1.0$ \\
\hline
\end{tabular}




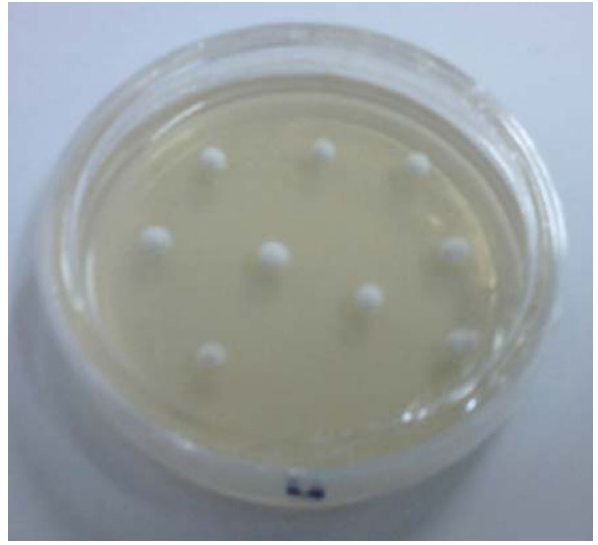

Figure 4.

$A B 4$ mycelial germination 48 hours after seeding in PDA.

However, at $4 \%$ silicate concentration, it was necessary a longer period (48 hours). At this concentration, then, the barrier property of silicate was evidenced, affecting the diffusion level of the encapsulated bioactive substance, in this case, the entomopathogenic fungus. In a study on entomopathogenic agents formulated in polymer systems containing alginate and citrus pectin, Moretini and Melo ${ }^{[17]}$ reported similar results, where the start of the growth of Coniothyrium minitans was verified 48 hours after seeding.

Finally, series 2 bead stability as a function of temperature is shown in Table 2.

The beads stored at low temperatures were more viable than those kept at room temperature regardless bentonite presence or concentration. This result shows the importance of temperature control for formulations containing fungal conidia, because they are very sensitive to external factors. Formulations containing silicate presented better results and AB4 showed the highest thermal stability even at room temperature. It reached an increasingly significant result when stored under refrigeration. Under this condition it maintained normal mycelial germination during the 7 months of the study. Similar results were found by Carneiro \& Gomes, ${ }^{[25]}$ encapsulating Paecilomyces lilacinus fungus in an alginate/bentonite matrix. The authors observed that viability was maintained even after 12 months of storage at $7^{\circ} \mathrm{C}$.

\section{Conclusion}

Fungal conidia are very sensitive to external factors (abiotic), thus, their encapsulation is necessary to ensure its marketing as bioinsecticide. Therefore, choosing the matrix material is essential and must be done according to its technological application. XRD analysis indicated that there was not intercalation of alginate in bentonite, but FTIR confirmed the interaction between both materials. The different tests used in the present study indicated that the addition of nanolayered silicate to the hydrophilic biopolymer seems to be useful, since it controlled the latter's high porosity.

Table 2.

Stability of encapsulated fungal conidia stored at different temperatures.

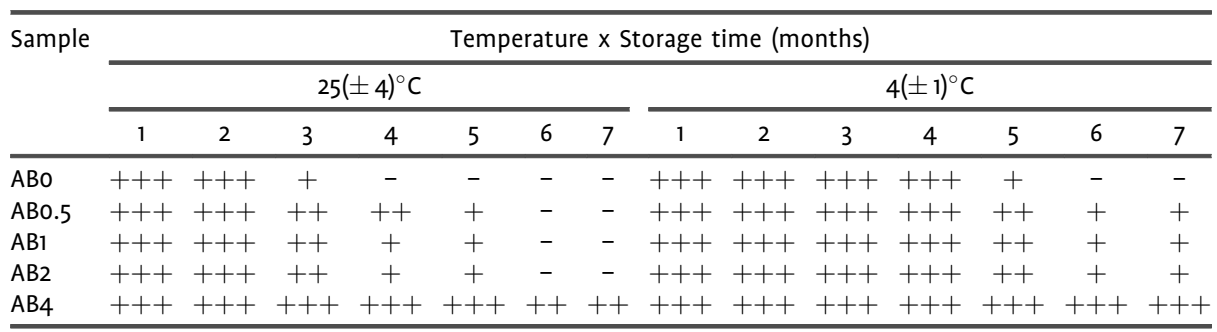

+++ indicates normal mycelial germination (compared with recently prepared beads).

++ indicates mycelial germination delayed approximately 24 hours.

+ indicates mycelial germination delayed approximately 72 hours and poor growth.

- indicates absence of mycelial growth. 
This was observed through the reduction of the average diameter of the obtained beads and swelling index and, depending on its concentration, a modification of fungus (bioactive substance) release and thermal stability of the formulation. Furthermore, the present study showed the matrix encapsulating property for fermented biomass with ability to incorporate conidia, even at high concentrations, without interfering in its germination process. Further studies will include the assessment of the effect of the matrix on fungus protection against other external factors, such as radiation, $\mathrm{pH}$ and moisture and biological tests on pest-insects.

Acknowledgements: The authors would like to thank the Brazilian Agricultural Research Corporation (EMBRAPA) and Foundation Research of the State of Alagoas (FAPEAL) for the financial support.

[1] B. Singh, D. K. Sharma, A. Gupta, J. Hazard. Mater., 2009, 161, 208-216.

[2] Environmental Protection Agency (EPA) - United States. Pesticides: Regulating Pesticides. Available at: <http://www.epa.gov/pesticides/biopesticides/ whatarebiopesticides.htm $>$.

[3] J. Calero, Y. F. Sánchez, R. Tórrez, E. Hernann, K. López, Universitas, 2008, 1, 27-30.

[4] Z. Wu, L. Guo, S. Qin, C. Li, J. Ind. Microbiol Biotechnol, 2012, 39, 317-327.

[5] D. K. Mishra, K. Behari, A. Sand, M. Yadav, Carbohydr. Polym., 2010, 80, 1147-1154.

[6] S. P. Lonkar, S. Morlat-Therias, N. Caperaa, F. Leroux, J. L. Gardette, R. P. Singh, Polymer, 2009, 50, 1505-1515. [7] Y. Ma, F. Shi, Z. Wang, M. Wu, J. Ma, C. Gao, Desalination, 2012, 286, 131-137.
[8] S. S. Ray, M. Bousmina, Prog. Mater. Sci., 2005, 50, 962-1079.

[9] S. Bourbigot, D. L. Vanderhart, J. W. Gilman, S. Bellayer, H. Stretz, D. R. Paul, Polymer, 2004, 45, 7627-7638.

[10] T. Hoang-Minh, T. L. Le, J. Kasbohm, R. Gieré, Appl. Clay Sci., 2010, 48, 349-357.

[11] B. Güerri-Agulló, S. Gómez-Vidal, L. Asensio, P. Barranco, L. V. Lopez-Llorca, Microsc Res. Tech., 2010, 73, 714-725.

[12] N. A. Maftei, A. Y. Ramos-Villarroel, A. L. Nicolau, O. Martínpbelloso, R. Soliva-Fortuny, Food Control, 2014, 41, 27-31.

[13] X. X. Wang, X. P. Ji, J. X. Li, N. O. Keyhani, M. G. Feng, S. H. Ying, Res. Microbiol., 2013, 164(5), 480-489. [14] L. Brügemann, E. K. E. Gerndt, Nucl. Instrum. Methods Phys. Res. A, 2004, 531(1-2), 292-301.

[15] V. Vijayan, S. D. Rao, E. Jayachandran, J. Anburaj, JITPS, 2010, 1(8), 320-328.

[16] O. A. Cavalcanti, G. Van den Mooter, I. CaramicoSoares, R. Kinget, Drug Dev. Ind. Pharm., 2002, 28, 157164.

[17] A. Moretine, I. S. Melo, Pesqui. Agropecu. Bras., 2007, 42(2), 155-161.

[18] R. T. Alves, R. P. Bateman, J. Gunn, C. Prior, S. Leather, Neotrop. Entomol., 2002, 31(1), 91-99.

[19] K. Mladenovska, O. Cruaud, P. Richomme, E. Belamie, R. S. Raicki, M. C. Venier-Julienne, E. Popovski, J. P. Benoit, K. Goracinova, Int. J. Pharm., 2007, 345, 59-69.

[20] B. D. Kevadiya, G. V. Joshi, H. A. Patel, P. G. Ingole, H. M. Mody, H. C. Bajaj, J. Biomater. Appl., 2010, 25. [21] H. A. Patel, R. S. Somani, H. C. Bajaj, R. V. Jasra, Appl. Clay. Sci., 2007, 35, 194-200.

[22] P. Thaned, P. Satit, AAPS Pharm. Sci. Tech., 2007, 8(3), E1-E7.

[23] P. Pasqualim, T. A. Culpi, G. T. A. Kaminski, M. T. Fin, D. G. B. Sasso, C. K. Costa, M. D. Miguel, G. M. Fujiwara, B. H. Rodrigues, S. M. W. Zanin, Visão Acadêmica, 2010, 11, 23-37.

[24] F. Cabellero, M. Foradada, M. Miñarro, P. PérezLozano, E. García-Montoya, J. R. Ticó, J. M. Suñé-Negre, International J. Pharm., 2014, 460, 181-188.

[25] R. M. D. G. Carneiro, C. B. Gomes, Nematol. Brasileira, 1997, 21, 85-92. 